Planetary Systems in the Universe - Observation, Formation and Evolution

Proceedings IAU Symposium No. 202, (C)2004 IAU

Alan Penny, Pawel Artymowicz, Anne-Marie Lagrange, 6 Sara Russell, eds.

\title{
Formation of the Planetary Sequence in a Highly Flattened Disk of Frequently Colliding Planetesimals
}

\author{
Evgeny Griv, Michael Gedalin, David Eichler \\ Dept. of Physics, Ben-Gurion University, Beer-Sheva 84105, Israel
}

Chi Yuan

Academia Sinica Institute of Astronomy, Taipei 11529, Taiwan

\begin{abstract}
The kinetic theory is used to study the evolution of the selfgravitating disk of planetesimals. The effects of frequent collisions between planetesimals are taken into account by using a Krook integral in the Boltzmann kinetic equation. It is shown that as a result of an aperiodic collision-dissipative instability of small gravity disturbances the disk is subdivided into numerous dense fragments. These can eventually condense into the planetary sequence.
\end{abstract}

Solar system formation is thought to start with dust particles settling to the central plane of a nebula to form a thin dust layer around the equatorial plane. During the early evolution of such a rapidly rotating disk it is believed that the dust particles coagulate into numerous kilometer-sized rocky bodies (planetesimals). See Taylor (1992) as a review of the problem.

In a swarm of planetesimals direct physical collisions inevitably become an important factor (Taylor 1992). One can suggest that planets (Mercury, Venus, ..., Neptune) accreted subsequently from a hierarchy of colliding planetesimals. (The combination of low mass with a highly inclined and eccentric orbit is a major reason for not according Pluto planetary status: observations point to the orbit of Neptune as the true outer boundary of the planetary system.)

Our principal idea is to regard the formation of the planetary system as a possible last stage in the formation of the self-gravitating, highly flattened solar nebula with frequent, almost elastic collisions between rocky planetesimals. We argue that a collision-dissipative collective instability of small-amplitude gravity perturbations developing in such a disk leads to the formation of an arrangement of dense aggregates. We speculate that subsequent substantial gravitational interaction between these dense aggregates circling the primordial sun would result in the formation of the planetary sequence. When cores of the giant planets reach a critical mass ( $\sim 10$ masses of the Earth) they begin to accrete the interplanetary gas.

The collision motion of an ensemble of identical planetesimals in the plane, in the frame of reference rotating with angular velocity $\Omega$, can be described by the Boltzmann kinetic equation

$$
\frac{\partial f}{\partial t}+\vec{v} \cdot \frac{\partial f}{\partial \vec{r}}-\frac{\partial \Phi}{\partial \vec{r}} \cdot \frac{\partial f}{\partial \vec{v}}=\left(\frac{\partial f}{\partial t}\right)_{\text {coll }},
$$

where $f(\vec{r}, \vec{v}, t)$ is the phase-space distribution function of planetesimals, $\Phi(\vec{r}, t)$ is the total gravitational potential, and $(\partial f / \partial t)_{\text {coll }}$ is the so-called collision in- 
tegral which takes into account effects due to the discrete-point nature of the gravitational charges and defines the change of $f$ arising from ordinary interparticle collisions. There are only elastic physical collisions, and momentum is conserved in collisions. There is no correlation in motion between the colliding species, that is, Boltzmann's hypothesis of molecular chaos is adopted.

The Poisson equation in a suitable form is $\nabla^{2} \Phi=4 \pi G \sigma \delta(z)$, where $\sigma(\vec{r}, t)$ is the surface mass density. The Boltzmann and Poisson equations, with the distribution function of planetesimals and appropriate boundary conditions, give a complete description of the problem for infinitesimally thin disk modes in a self-consistent field problem. In the present study, this system of equations is simplified by considering the lowest WKB approximation (in the limit $\nu_{\mathrm{c}} \gg \Omega$, where $\nu_{\mathrm{c}}$ is the frequency of collisions between planetesimals); this is accurate for short wave perturbations only, but qualitatively correct even for perturbations with a longer wavelength, of the order of the system radius.

As is known, the Boltzmann equation is nearly intractable because of the complicated collision integral. This integral can be approximated in various ways. In this work we use the simplified kinetic model when the exact integral $(\partial f / \partial t)_{\text {coll }}$ is replaced by a phenomenological term in the form of a Krook model (Lifshitz \& Pitaevskii 1981; Griv \& Peter 1996; see also Griv et al. 1999).

Perturbations in the gravitational field cause perturbations to the planetesimal distribution function. In the linear approximation, one can therefore write $f(\vec{r}, \vec{v}, t)=f_{0}(r, \vec{v})+f_{1}(\vec{r}, \vec{v}, t)$, where $\left|f_{1}\right| \ll f_{0}$ and $f_{1}$ is a function rapidly oscillating in space and time. We consider zeroth order disk inhomogeneity in the $r$-direction only. Initially the disk is in equilibrium, $\partial f_{0} / \partial t=0$. The function $f_{0}$ describes the rotating "background" against which small perturbations develop. If an initial perturbation grows, the system is called unstable.

As a result of this study, the dispersion relation connecting the Dopplershifted wavefrequency $\omega_{*}$ and the wavenumber $k$ of perturbations is found:

$$
1-\frac{2 \pi G \sigma_{0}}{|k| c^{2}}\left\{1-\frac{\sqrt{\pi} g}{\sqrt{2} k c} \exp \left(\frac{g^{2}}{2 k^{2} c^{2}}\right)\left[1-\operatorname{erf}\left(\frac{g}{\sqrt{2} k c}\right)\right]\right\}=0
$$

where $\sigma_{0}(r)$ is the equilibrium surface density, $c$ is the dispersion of random velocities of planetesimals ("temperature"), $g=i\left(\gamma+\nu_{\mathrm{c}}\right)$, and $\operatorname{erf}(\beta)$ denotes the error function. The wavefrequency is represented in the form $\omega \equiv i \gamma$, where $\gamma$ is real and positive (cf. Binney \& Tremaine 1987, p. 292).

A numerical solution of the dispersion relation is shown in Figure 1. As follows from this solution, the dispersion relation gives the condition for an aperiodic collision-dissipative instability $(\gamma>0)$ :

$$
\lambda>\lambda_{\text {crit }} \approx c^{2} / G \sigma_{0} \text { and } \lambda=2 \pi / k,
$$

which is just the familiar Jeans criterion for a nonrotating medium and $\lambda_{\text {crit }}$ is the ordinary Jeans length (Binney \& Tremaine 1987). Frequent collisions thus remove the rotational stabilization in a flat system. Thus, the theory predicts that a gravitating disk with frequent interparticle collisions is aperiodically unstable for perturbations with wavelengths greater than the Jeans length.

At the wavelength $k_{*} \approx G \sigma_{0} / c^{2}$ the growth rate of the instability is maximum (Fig. 1). It means that of all harmonics of initial perturbation, one 


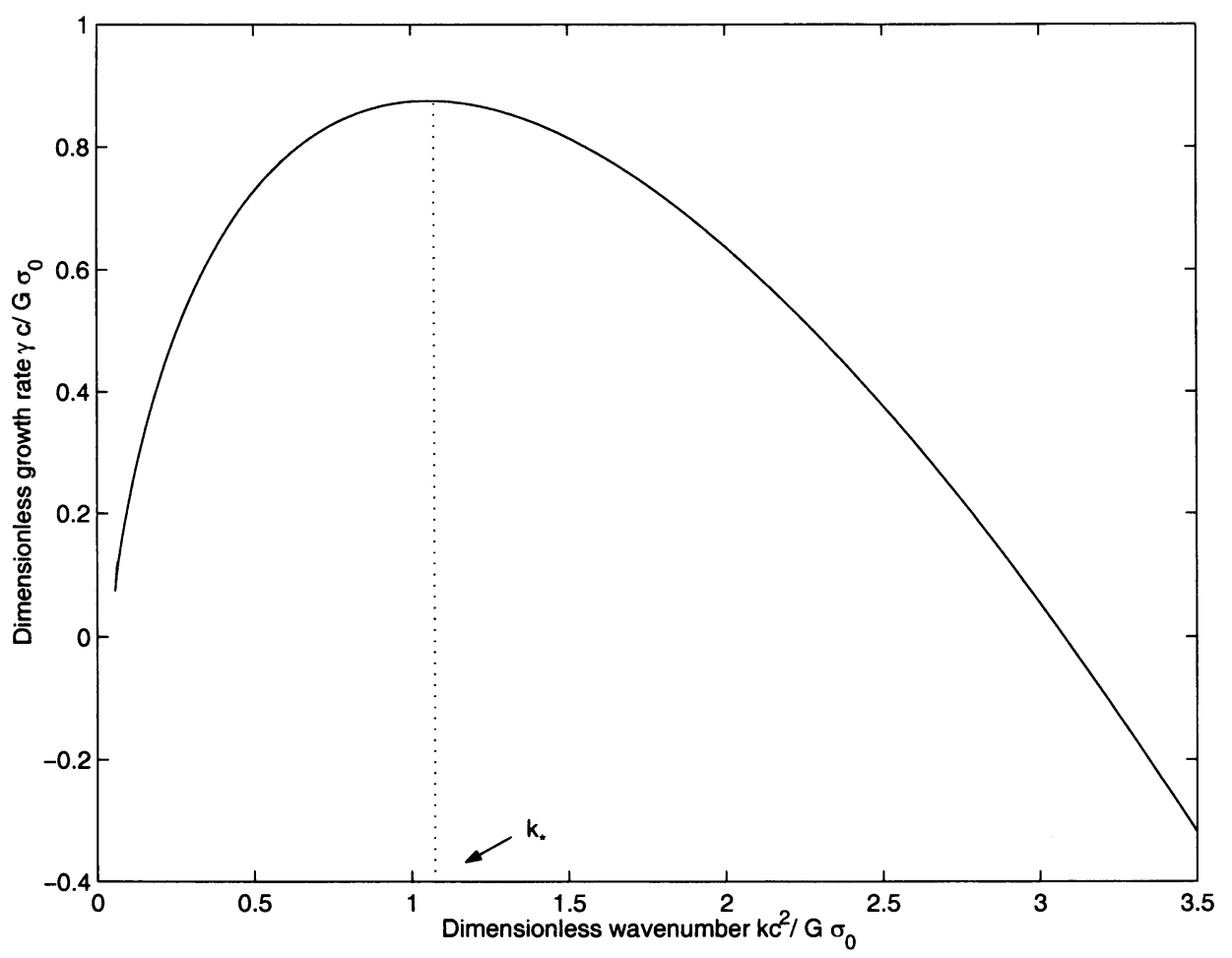

Figure 1. The dispersion relation for reasonable parameters of a twodimensional system of frequently colliding planetesimals.

perturbation with the maximum of the growth rate and with $k_{*}$ will be formed asymptotically in time. The numerous condensations with typical dimensions and distances between them $\lambda_{*}=2 \pi / k_{*}$ that arise will remain localized in space and grow, because the instability will be an aperiodic.

Acknowledgments. Support from the Israel Science Foundation and the Israeli Ministry of Immigrant Absorption is acknowledged.

\section{References}

Binney, J., \& Tremaine, S. 1987, Galactic Dynamics (Princeton University Press: Princeton, NJ)

Griv, E., \& Peter, W. 1996, ApJ, 469, 103

Griv, E., Rosenstein, B., Gedalin, M., \& Eichler, D. 1999, A\&A, 347, 821

Lifshitz, E. M., \& Pitaevskii, L. P. 1981, Physical Kinetics (Oxford: New York)

Taylor, S. R. 1992, Solar System Evolution (Cambridge University Press: Cambridge) 